MR OLIVER PEACOCK (Orcid ID : 0000-0002-1645-4301)

DR NICHOLAS SMITH (Orcid ID : 0000-0002-0122-2719)

DR PEADAR SEAN WATERS (Orcid ID : 0000-0003-2947-9206)

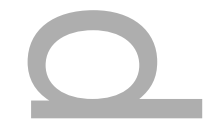

Article type : Original Article

CDI-00594-2019.R2

Original Article

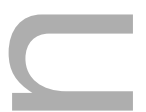

\title{
Outcomes of extended radical resections for locally advanced and recurrent pelvic malignancy involving the aortoiliac axis
}

Oliver Peacock PhD FRCS ${ }^{1 *}$, Nicholas Smith FRACS ${ }^{1 *}$, Peadar S Waters MD FRCS ${ }^{1}$, Francis Cheung MD, Jacob J McCormick FRACS ${ }^{1}$, Satish K Warrier MS FRACS ${ }^{1}$, Timothy Wagner ${ }^{2}$ FRACS, Alexander G Heriot ${ }^{1}$ MD FRACS

Colorectal Surgery Unit, Peter MacCallum Cancer Centre, Melbourne, Australia ${ }^{1}$.

Vascular Surgery Unit, Royal Melbourne Hospital, Melbourne, Australia².

*Combined first authors

Corresponding Author \& Reprints:

Oliver Peacock

Colorectal Surgery Unit, Peter MacCallum Cancer Centre, 305 Grattan Street, Melbourne, Victoria 3000, Australia Telephone Number: 0385595000, Fax Number: 0385597379

Email: oliver.peacock@nhs.net

This is the author manuscript accepted for publication and has undergone full peer review but has not been through the copyediting, typesetting, pagination and proofreading process, which may lead to differences between this version and the Version of Record. Please cite this article as doi: 10.1111/CODI.14969

This article is protected by copyright. All rights reserved 
Conflict of Interest and Source of Funding: None

No sources of support

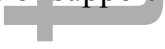

Word Count: 1,859 words (Excluding abstract, references, tables and figures)

References: 26
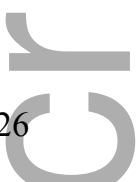

Short running head: Pelvic vascular exenteration outcomes

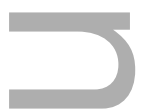

What does this paper add to the literature?: This series adds increasing evidence that good outcomes can be achieved for extended radical pelvic resections in locally advanced and recurrent pelvic malignancies with aortoiliac axis involvement. A coordinated approach in specialist centres demonstrates this is safe and feasible, offering low major complication rates.

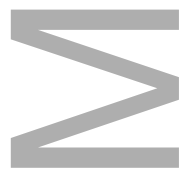

AIM: Currently, there is no clear consensus on the role of extended pelvic resections for locally advanced or recurrent disease involving major vascular structures. The aims of this study were to report the outcomes of consecutive patients undergoing extended resections for pelvic malignancy involving the aortoiliac axis.

METHODS: Prospective data on patients having extended radical resections for locally advanced or recurrent pelvic malignancies, with aortoiliac axis involvement, requiring en-bloc vascular resection and reconstruction, at a single institution between 2014 and 2018 were collected.

RESULTS: Eleven patients were included (median age 60 years: range 31-69 years; 7 females). The majority required resection of both arterial and venous systems $(n=8)$, and the technique for vascular reconstruction was either interposition grafts or femoral-femoral crossover grafts. The median operative time was 510 mins (range 330-960 mins). Clear resection margins (R0) were achieved in 9 patients. The median length of stay was 25 days (range 7-83 days). Seven patients did not suffer an early complication. There was one serious complication (Clavien-Dindo $\geq 3$ ), an arterial graft occlusion secondary to thrombus in the immediate postoperative period, requiring a return to theatre and thrombectomy. The median length of follow-up in this study was 22 months (range 4-58 months). 
CONCLUSION: This series demonstrates that en-bloc major vascular resection and reconstruction can be performed safely and achieve clear resection margins in selected patients with locally advanced or recurrent pelvic malignancy at specialist surgery centres.

KEYWORDS: Pelvic exenteration, malignancy, 30-day morbidity \& mortality, vascular reconstruction, vascular resection

\section{INTRODUCTION:}

Pelvic exenteration is now an established procedure for locally advanced and recurrent pelvic malignancies. Complete surgical resection offers the potential for cure, providing a negative resection margin is achieved. Reluctance to pursue surgical resection for these cases has been based on the significant morbidity, limitations in achieving clear margins and the impact on prognosis[1,2].

Traditionally, pelvic sidewall involvement has been considered a contraindication for surgery, with the lateral compartment typically posing the most challenge[3] due to the bony limitations of the pelvis and the major neurovascular components[4, 5]. Previously, lateral pelvic sidewall tumour involvement had a worse prognosis than tumours involving the other compartments of the pelvis, due to the difficulty of achieving a clear resection[6, 7]. There is also currently no clear consensus on the role of extended resections for locally advanced or recurrent disease involving major vascular structures.

However, with improved surgical technique, surgical units with exenterative experience have expanded the boundaries of resectability and redefined what constitutes resectable disease[3]. Moreover, recent small series for vascular resections as part of pelvic exenterations have presented good outcomes[3, 8, 9], but the literature remains limited to a small number of institutions.

The aims of this study were to report the clinical and pathological outcomes of consecutive patients undergoing extended radical pelvic resections for locally advanced or recurrent pelvic malignancy involving the aortoiliac axis.

\section{METHODS:}

A retrospective review of a prospectively maintained database was undertaken to assess the clinical and pathological outcome of patients who have undergone extended radical pelvic resections for locally advanced or recurrent pelvic malignancies with aortoiliac axis involvement. The primary objectives were to identify the complications and pathological outcomes following aortoiliac resection and reconstruction as part of the extended radical pelvic resections. One tertiary referral centre, Peter MacCallum Cancer Centre (Melbourne, Australia), with specialist experience in the surgical management of advanced and recurrent pelvic tumours was included. All patients were routinely discussed at a dedicated pelvic exenterative surgery multidisciplinary meeting, identifying those with aortoiliac axis involvement requiring en-bloc resection and reconstruction. The diagnosis of locally advanced or recurrent pelvic tumour was based on preoperative radiological imaging (PETCT and MRI) and clinical assessment. All patients were reviewed clinically by a vascular surgeon and subsequent investigations were conducted as required. Patient demographics (age, sex, ASA), comorbidities, 
neoadjuvant \& adjuvant therapies, operative details including type of vascular resection and reconstruction, histopathological staging, morbidity, mortality, length of hospital stay, local and distant recurrence at follow-up were recorded.

The technique for extended radical pelvic resection at our institution has previously been described[6, 10]. Operations were considered extended radical when the primary organ and at least one of the surrounding organs was removed en-bloc[6]. The operative procedure for the vascular resection and reconstruction was very similar to previously published studies[3, 8,11$]$.

Histopathological evaluation defined margin status as R0 resection (margins clear of disease), R1 resection (presence of microscopic residual disease at the margins) and R2 resection (the presence of macroscopic residual disease at the margins).

Complications occurring within 30-days postoperatively or during the inpatient care for the index operation were graded according to the Clavien-Dindo classification system for surgical complications[12]. A major complication was defined as Grade III or IV of the Clavien-Dindo classification. Inpatient mortality was defined as a death occurring within 30-days of the index procedure.

No statistical analysis was performed for this descriptive study.

Specific considerations for the vascular reconstruction:

A CT angiogram, with a portal venous phase, is performed preoperatively on any patient being considered for vascular resection beyond the internal iliac vessels to facilitate planning of any operative strategy. All synthetic vascular grafts (Dacron ${ }^{\circledR}$ or PTFE) are soaked in Rifampicin prior to performing the vascular reconstruction and prophylactic antibiotics are continued for 5 days following surgery.. Since 2017, a pre-emptive arterial and/or venous femoral-femoral crossover reconstruction now occurs approximately 4 weeks prior to the extended radical resection, to further reduce the risk of graft infection from the contaminated field. The surgeon must be vigilant at the inferior aspect of the midline incision during the extended radical resection to avoid inadvertent damage to the graft. All patients are discharged on therapeutic enoxaparin for anticoagulation and are converted to novel oral anticoagulants (NOACs) following completion of any required systemic therapy postoperatively. Routine vascular ultrasound surveillance occurs at 6-weeks, 3-months, 6-months and at 1-year postoperatively to monitor the patency of the vascular grafts.

\section{RESULTS:}

Eleven consecutive patients underwent vascular resection and reconstruction of the aortoiliac axis as part of an extended radical pelvic resection for locally advanced or recurrent pelvic malignancy with curative intent at a single institution between June 2014 and November 2018. The median age for this cohort of patients was 60 years (range 31-69 years) and the majority were female patients $(n=7 ; 63.6 \%)$. Patient demographics and clinical characteristics are outlined in Table 1. The main pathology was recurrent rectal adenocarcinoma $(\mathrm{n}=8$, $72.7 \%$ ). The median operative time was 510 mins (range 330-960 mins). The median length of follow-up in this study was 22 months (range 4-58 months). 
The extent of vascular resection and reconstruction is outlined in Table 2. The majority of patients required resection of both arterial and venous systems $(n=8)$, and synthetic grafts were the commonest reconstruction method. The technique for vascular reconstruction was either interposition grafts or femoral-femoral crossover grafts (Table 2). Clear resection margins (R0) were achieved in 9 patients $(81.8 \%)$. One patient required excision of the infra-renal aorta and left iliac vessels due to direct tumour invasion. This resected portion had signet ring adenocarcinoma present, but superior and inferior margins were clear and no presence of luminal surface involvement. Two further patients had microscopic invasion of adenocarcinoma into the vessel, whilst the remaining patients had tumour abutting the vessel or extensive fibrosis following radiotherapy. The extent of the resections, based on the seven intra-pelvic compartments[13] and organs resected, to attain clear margins are outlined in Table 3.

There was no postoperative mortality. The median length of stay was 25 days (range 7-83 days). Seven patients did not suffer any early complications. There were no graft infections. The remaining four patients suffered one or more early complications and these are shown in Table 4. There was only one serious complication in this series, an arterial graft occlusion secondary to thrombus in the immediate postoperative period, requiring a return to theatre and thrombectomy. During routine surveillance, one patient developed an asymptomatic venous graft thrombosis and continued anticoagulation therapy.

Currently, 9 patients are alive following vascular resection and reconstruction for locally advanced or recurrent pelvic malignancy. Two patients have subsequently developed local recurrence 15 and 25 months respectively following surgery. There have been two deaths in this series, all due to metastatic disease. One patient developed local recurrence and subsequently developed distal metastases and died 14 months following surgery. The other patient developed distant metastases and died 22 months after surgery.

\section{DISCUSSION:}

This series of patients undergoing radical surgery for locally advanced and recurrent pelvic malignancy involving the aortoiliac axis demonstrates that clear margins $(81.8 \%$; $=9)$ can be achieved and is associated with low major morbidity $(9 \% ; n=1)$.

Previously, pelvic malignancies involving the lateral pelvic side wall were associated with higher positive margin rates and poorer survival compared with anterior, central or posterior compartment tumours[6, 14, 15]. The Beyond Total Mesorectal Excision (TME) Collaborative developed a consensus statement that outlined encasement of the external or common iliac vessels requiring en-bloc resection and reconstruction to be a relative contraindication to surgery by $78 \%$ of the experts[16]. Moreover, other reviews have considered common or external iliac vessel involvement to be an absolute contraindication to surgery[17, 18]. However, recent studies have also shown acceptable morbidity and R0 resection rates ranging between $38 \%$ to $69 \%[3,8$, $9,19]$, following resection and reconstruction of the aortoiliac axis. This current study supports the small, but growing body of evidence, that tumours previously deemed unresectable due to vascular involvement, could be curatively resected in selected patients[20].

The surgical community has been slow to adopt pelvic exenteration for advanced malignancy, undoubtedly owing to concerns regarding high morbidity and mortality rates based on historical data[21, 22], and accessibility to surgery for those with potentially curative local recurrence remains a concern[23]. Advances in 
imaging techniques, development of a multi-disciplinary approach and improved surgical technique and technology has redefined resectability $[9,22]$. Although locally advanced tumours are not necessarily modifiable, they are preoperatively identifiable, with pelvic MRI able to identify patients at high risk of positive margins[24] and is the key imaging modality in guiding assessment and planning[4]. Although MRI has high accuracy in determining invasion into pelvic structures, the sensitivity of MRI is lower when assessing tumour invasion in the lateral pelvic sidewall[13,25]. This difficulty in assessing the extent of lateral pelvic sidewall invasion, highlights that patients require cross-disciplinary skills and multi-speciality collaboration with experience in managing these complex cases to achieve the best outcomes. These cases necessitate institutions providing state of the art diagnostic, therapeutic and interventional radiology, advanced intensive care capabilities and strong blood-bank support[26]. Computed tomography combined with positron emission tomography (CT-PET) imaging is also part of the staging process for every patient to evaluate local and assess for any distant disease. The multi-disciplinary specialists must also have an interest in extended pelvic resections and reconstruction, with such factors arguing in favour of centralisation to provide a carefully coordinated team approach[26]. Therefore, locally advanced or recurrent pelvic malignancy patients with aortoiliac axis involvement require evaluation by an experienced MDT exenteration centre with defined management protocols to assess whether the disease is potentially curative[11]. This detailed discussion facilitates the selection of appropriate patients and the meticulous planning of the surgical intervention with a specialist vascular surgeon.

If a clear dissection plane cannot be delineated on preoperative imaging between vascular structures and the tumour, then an en-bloc resection is advocated. During our initial experience, vascular resection and reconstruction was performed concomitantly. However, this resulted in longer operating times, but more importantly, longer ischaemic time, in order to facilitate mobilisation of the tumour requiring early division of the vessel proximally. Also, there was concern regarding in-situ reconstruction with an artificial vascular graft (despite soaked in Rifampicin) in the presence of a bowel resection leading to a contaminated field. For these reasons, the technique in our institution has evolved. A pre-emptive arterial and venous femoral-femoral crossover graft is performed approximately 4 weeks prior and the patency of the grafts ensured before the extended radical pelvic resection with en-bloc vascular resection is performed to reduce some of the risks outlined above.

There are limitations to this study, including the small sample size, retrospective study design, heterogeneity of the patient population and inherent selection bias, which need to be considered. However, due to the limited data available, this study adds to the small, but growing evidence that resection and reconstruction of the aortoiliac axis for advanced pelvic malignancy is safe and feasible.

Conclusion: This series demonstrates that clear resection margins can be achieved in selected patients with enbloc major vascular resections within a specialised centre for exenterative surgery and vascular reconstruction can be performed safely.

Acknowledgements:

Nil

Disclosures:

This article is protected by copyright. All rights reserved 


\section{REFERENCES:}

1. Boyle KM, Sagar PM, Chalmers AG, Sebag-Montefiore D, Cairns A, Eardley I. (2005), Surgery for locally recurrent rectal cancer. Dis Colon Rectum, 48: 929-937.

2. Bouchard P, Efron J. (2010), Management of recurrent rectal cancer. Ann Surg Oncol, 17: 1343-1356.

3. Brown KG, Koh CE, Solomon MJ, Qasabian R, Robinson D, Dubenec S. (2015), Outcomes After En Bloc Iliac Vessel Excision and Reconstruction During Pelvic Exenteration. Dis Colon Rectum, 58: 850-856.

4. Chew MH, Brown WE, Masya L, Harrison JD, Myers E, Solomon MJ. (2013), Clinical, MRI, and PET-CT criteria used by surgeons to determine suitability for pelvic exenteration surgery for recurrent rectal cancers: a Delphi study. Dis Colon Rectum, 56: 717-725.

5. Sagar PM. (2006), Extended surgery for local recurrence and advanced rectal cancer. Colorectal Dis, 8 Suppl 3: 43-46.

6. Heriot AG, Byrne CM, Lee P, Dobbs B, Tilney H, Solomon MJ, et al. (2008), Extended radical resection: the choice for locally recurrent rectal cancer. Dis Colon Rectum, 51: 284-291.

7. Bhangu A, Ali SM, Brown G, Nicholls RJ, Tekkis P. (2014), Indications and outcome of pelvic exenteration for locally advanced primary and recurrent rectal cancer. Ann Surg, 259: 315-322.

8. Abdelsattar ZM, Mathis KL, Colibaseanu DT, Merchea A, Bower TC, Larson DW, et al. (2013), Surgery for locally advanced recurrent colorectal cancer involving the aortoiliac axis: can we achieve R0 resection and long-term survival? Dis Colon Rectum, 56: 711-716.

9. Austin KK, Solomon MJ. (2009), Pelvic exenteration with en bloc iliac vessel resection for lateral pelvic wall involvement. Dis Colon Rectum, 52: 1223-1233.

10. Peacock O, Waters PS, Bressel M, Lynch AC, Wakeman C, Eglinton T, et al. (2019), Prognostic factors and patterns of failure after surgery for T4 rectal cancer in the beyond total mesorectal excision era. $\mathrm{Br} J$ Surg, doi: 10. 1002/bjs. 11242.

11. Wilson K, Waters PS, Peacock O, Heriot AG, Wagner T, Warrier SK. (2019), Multivisceral, vascular and nodal resection for recurrent rectal cancer involving the left renal tract, left pelvic side wall and abdominal aorta. ANZ J Surg, doi: 10. 1111/ans. 15281.

12. Dindo D, Demartines N, Clavien PA. (2004), Classification of surgical complications: a new proposal with evaluation in a cohort of 6336 patients and results of a survey. Ann Surg, 240: 205-213.

13. Georgiou PA, Tekkis PP, Constantinides VA, Patel U, Goldin RD, Darzi AW, et al. (2013), Diagnostic accuracy and value of magnetic resonance imaging (MRI) in planning exenterative pelvic surgery for advanced colorectal cancer. Eur J Cancer, 49: 72-81.

14. Yamada K, Ishizawa T, Niwa K, Chuman Y, Aikou T. (2002), Pelvic exenteration and sacral resection for locally advanced primary and recurrent rectal cancer. Dis Colon Rectum, 45: 1078-1084.

15. Moore HG, Shoup M, Riedel E, Minsky BD, Alektiar KM, Ercolani M, et al. (2004), Colorectal cancer pelvic recurrences: determinants of resectability. Dis Colon Rectum, 47: 1599-1606. 
16. Beyond TME Consensus. (2013), Consensus statement on the multidisciplinary management of patients with recurrent and primary rectal cancer beyond total mesorectal excision planes. Br J Surg, 100: 10091014.

17. Mirnezami AH, Sagar PM, Kavanagh D, Witherspoon P, Lee P, Winter D. (2010), Clinical algorithms for the surgical management of locally recurrent rectal cancer. Dis Colon Rectum, 53: 1248-1257.

18. Pawlik TM, Skibber JM, Rodriguez-Bigas MA. (2006), Pelvic exenteration for advanced pelvic malignancies. Ann Surg Oncol, 13: 612-623.

19. Solomon MJ, Brown KG, Koh CE, Lee P, Austin KK, Masya L. (2015), Lateral pelvic compartment excision during pelvic exenteration. Br J Surg, 102: 1710-1717.

20. Harji DP, Griffiths B, McArthur DR, Sagar PM. (2013), Surgery for recurrent rectal cancer: higher and wider? Colorectal Dis, 15: 139-145.

21. Brown KGM, Solomon MJ, Koh CE. (2017), Pelvic Exenteration Surgery: The Evolution of Radical Surgical Techniques for Advanced and Recurrent Pelvic Malignancy. Dis Colon Rectum, 60: 745-754.

22. Brown KGM, Solomon MJ. (2018), Progress and future direction in the management of advanced colorectal cancer. BrJ Surg, 105: 615-617.

23. Harji DP, Griffiths B, McArthur DR, Sagar PM. (2012), Current UK management of locally recurrent rectal cancer. Colorectal Dis, 14: 1479-1482.

24. Mercury Study Group. (2006), Diagnostic accuracy of preoperative magnetic resonance imaging in predicting curative resection of rectal cancer: prospective observational study. BMJ, 333: 779 .

25. Dresen RC, Kusters M, Daniels-Gooszen AW, Cappendijk VC, Nieuwenhuijzen GA, Kessels AG, et al. (2010), Absence of tumor invasion into pelvic structures in locally recurrent rectal cancer: prediction with preoperative MR imaging. Radiology, 256: 143-150.

26. Madoff RD. (2006), Extended resections for advanced rectal cancer. Br J Surg, 93: 1311-1312.

Table 1: Baseline patient characteristics

\begin{tabular}{|l|l|}
\hline \multicolumn{1}{|c|}{ Characteristic } & \multicolumn{1}{c|}{ Total } \\
\hline Sex & $4(36.4 \%)$ \\
Male & $7(63.6 \%)$ \\
Female & $6(54.5 \%)$ \\
\hline Age & $5(45.5 \%)$ \\
$<60$ & 0 \\
$>60$ & 5 \\
\hline ASA & 6 \\
1 & 0 \\
\hline 3 & \\
\hline Cancer presentation & 0 \\
\hline
\end{tabular}




\begin{tabular}{|l|l|}
\hline \multicolumn{1}{|c|}{ Rectal: } & 9 \\
Recurrent & 1 \\
Cervical & 8 \\
Uterine & 1 \\
\hline Radiotherapy & 1 \\
Yes & 11 \\
No & 0 \\
\hline Chemotherapy & \\
Yes & 11 \\
No & 0 \\
\hline Previous thromboembolic disease & \\
Yes & 2 \\
No & 9 \\
\hline Preoperative anticoagulation & \\
Yes & 2 \\
No & 9 \\
\hline Margin Status & \\
R0 & 9 \\
R1 & 25 days (range 7-83) \\
\hline Median blood loss & \\
\hline Median operative time & \\
\hline Median length of stay & \\
\hline ASA: Amesitres (range 0.5-3) \\
\hline
\end{tabular}

ASA: American Society of Anaesthesiologists

Table 2: Details of the vascular resection and reconstruction

\begin{tabular}{|l|l|l|}
\hline & \multicolumn{1}{|c|}{$\begin{array}{c}\text { Arterial } \\
(\mathrm{n}=10)\end{array}$} & \multicolumn{1}{c|}{$\begin{array}{c}\text { Venous } \\
(\mathrm{n}=9)\end{array}$} \\
\hline Resection & CIA/EIA/IIA 4 & CIV/EIV/IIV 6 \\
EIA/IIA 3 & \\
& EIA 2 3 \\
& CIA/EIA/IIA/Aorta 1 & \\
\hline
\end{tabular}




\begin{tabular}{|l|l|l|}
\hline Reconstruction method & Interposition graft 3 & Interposition graft 4 \\
& Femoral-femoral crossover graft 6 & Femoral-femoral crossover graft 5 \\
& Both 1 & \\
\hline Reconstruction technique & & 1 \\
Autologous (LSV) & 1 & \\
Synthetic & 9 & 0 \\
Dacron ${ }^{\circledR}$ & 0 & 8 \\
PTFE & & \\
\hline
\end{tabular}

CIA: common iliac artery; EIA: external iliac artery; IIA: internal iliac artery; CIV: common iliac vein; EIV: external iliac vein; IIV: internal iliac vein; LSV: long saphenous vein; PTFE: polytetrafluoroethylene.

Arterial and venous resection: 8 patients;

Arterial resection alone: 2 patients;

Venous resection alone: 1 patient.

Table 3: Extent of the resection

\begin{tabular}{|l|l|}
\hline Compartments Resected & Total \\
\hline Lateral \& Anterior above peritoneal reflection & 4 \\
\hline Lateral, Anterior above \& below peritoneal reflection & 4 \\
\hline $\begin{array}{l}\text { Lateral, Central \& Anterior above peritoneal } \\
\text { reflection }\end{array}$ & 1 \\
\hline $\begin{array}{l}\text { Lateral, Central, Anterior above \& below peritoneal } \\
\text { reflection }\end{array}$ & 1 \\
\hline $\begin{array}{l}\text { Lateral, Central, Anterior above \& below peritoneal } \\
\text { reflection \& Inferior }\end{array}$ & 1 \\
\hline
\end{tabular}

The type of extended radical resection is outlined using the seven intrapelvic compartments, previously defined by Georgiou et al[13], which demonstrates the organs that are included in each compartment.

Table 4: Postoperative morbidity. Four patients suffered one or more complications.

\begin{tabular}{|l|l|l|}
\hline Type of Complication & Total & Clavien-Dindo Grade \\
\hline Ileus & 1 & I \\
\hline Lymphocele & 4 & II \\
\hline Pneumonia & 1 & II \\
\hline
\end{tabular}




\begin{tabular}{|l|l|l|}
\hline NSTEMI & 1 & II \\
\hline Arterial graft occlusion & 1 & III \\
\hline
\end{tabular}

NSTEMI: Non-ST elevation myocardial infarction

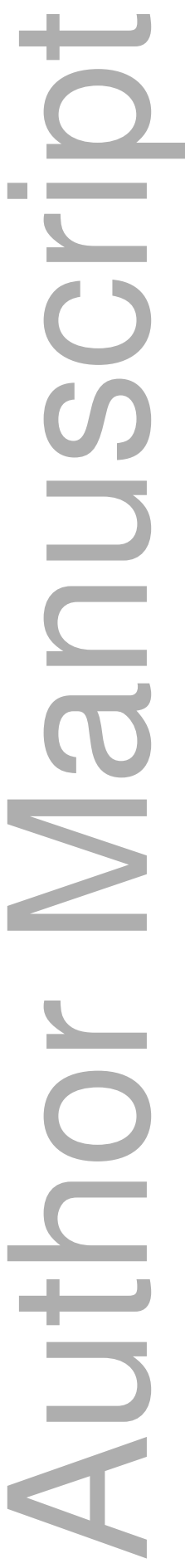




\section{University Library}

\section{- M M N E R VA A gateway to Melbourne's research publications}

Minerva Access is the Institutional Repository of The University of Melbourne

Author/s:

Peacock, O;Smith, N;Waters, PS;Cheung, F;McCormick, JJ;Warrier, SK;Wagner, T;Heriot, AG

Title:

Outcomes of extended radical resections for locally advanced and recurrent pelvic malignancy involving the aortoiliac axis

Date:

2020-02-11

\section{Citation:}

Peacock, O., Smith, N., Waters, P. S., Cheung, F., McCormick, J. J., Warrier, S. K., Wagner, T. \& Heriot, A. G. (2020). Outcomes of extended radical resections for locally advanced and recurrent pelvic malignancy involving the aortoiliac axis. COLORECTAL DISEASE, 22 (7), pp.818-823. https://doi.org/10.1111/codi.14969.

Persistent Link:

http://hdl.handle.net/11343/275394 